\title{
IRREDUCIBILITY OF HECKE POLYNOMIALS
}

\author{
SRinath Baba AND M. Ram Murty
}

\begin{abstract}
In this note, we show that if the characteristic polynomial of some Hecke operator $T_{n}$ acting on the space of weight $k$ cusp forms for the group $\mathrm{SL}_{2}(\mathbb{Z})$ is irreducible, then the same holds for $T_{p}$, where $p$ runs through a density one set of primes. This proves that if Maeda's conjecture is true for some $T_{n}$, then it is true for $T_{p}$ for almost all primes $p$.
\end{abstract}

\section{Introduction}

Let $V$ be the $d$-dimensional space of weight $k$ cusp forms $S_{k}\left(\mathrm{SL}_{2}(\mathbb{Z})\right)$, and $T_{n}$ the $n^{\text {th }}$ Hecke operator on $V$. Let $T_{n}(x)$ denote the characteristic polynomial of $T_{n}$. By the theory of eigenforms, it is well known that $T_{n}(x) \in \mathbb{Z}[x]$ and is monic. Maeda [8] conjectured that for some $n, T_{n}(x)$ is irreducible with Galois group $S_{d}$, where $S_{d}$ is the symmetric group on $d$ symbols. A popular extension of this conjecture, called Maeda's conjecture states that for every $n, T_{n}(x)$ is irreducible with Galois group $S_{d}$.

Recent progress related to Maeda's conjecture has been in two different directions. The first is to verify the conjecture for $T_{2}(x)$ for different weights $k$ ([1], [12]), and the second has been to show irreducibility of $T_{p}(x)$ assuming the irreducibility of $T_{q}(x)$ for some $q$. In [2], it is shown using the trace formula in characteristic $p$ that if some $T_{q}(x)$ satisfies Maeda's conjecture, then the same holds for $T_{p}(x)$ for $p$ in a set of primes of density $5 / 6$. Combining this with computer computations, K. James and D. Farmer have shown in [3] that if $T_{q}(x)$ satisfies Maeda's conjecture for some $q$, then the same holds for $T_{p}(x)$ for primes $p \leq 2000$.

The purpose of this note is to extend the work of both [2] and [3]. As opposed to the mod- $p$ versions of the trace formula used by [2], we study Frobenius distributions and Galois representations of Hecke eigenforms to show that

Theorem 1.1. If $T_{q}(x)$ is irreducible for some prime $q$, then

$$
\sharp\left\{p \leq x ; T_{p}(x) \text { is reducible }\right\} \ll x /(\log x)^{1+\delta},
$$

for some $\delta>0$.

In addition, we show the following:

Theorem 1.2. If $T_{q}(x)$ is irreducible with Galois group $S_{d}$ for some prime $q$, then the same holds for $T_{n}(x)$ for $n \leq d$.

Received October 16, 2001. 
The argument is based on the existence of a so called Miller basis for $V$, and uses linear algebra in combination with the Chebotarev density theorem and theorems of Deligne and Serre on the existence of certain $\ell$-adic representations attached to Hecke eigenforms.

\section{Preliminaries}

For any cusp form $g$, let $g=\sum_{i=1}^{\infty} a_{n}(g) q^{n}$ denote its Fourier expansion at the cusp at $\infty$. Let $K(g)=\mathbb{Q}\left(\left\{a_{i}(g)\right\}_{i=1}^{\infty}\right)$. Let $f_{1}, \ldots f_{d}$ denote a basis of normalised Hecke eigenforms for $V$. For any $i, 1 \leq i \leq d$, it is known that $K\left(f_{i}\right)$ is a number field of finite degree, and that the $a_{n}$ are all algebraic integers. Since the $f_{i}$ are simultaneous eigenvectors of all the Hecke operators, we know that $T_{n}(x)=\prod_{i=1}^{d}\left(x-a_{n}\left(f_{i}\right)\right)$.

Lemma 2.1. Suppose $T_{q}(x)$ is irreducible, where $q$ is a prime. Then $K\left(f_{i}\right)=$ $\mathbb{Q}\left(a_{q}\left(f_{i}\right)\right)$.

Proof. Let $h_{1}, \ldots h_{d}$ be the Miller basis for $V$. This basis is characterised by the property that $K\left(h_{i}\right)=\mathbb{Q}$ for every $i$, and $a_{i}\left(h_{j}\right)=\delta_{i, j}$, for $1 \leq i, j \leq d$, where $\delta_{i, j}=1$ if $i=j$ and 0 otherwise (see [6]). Let $f$ be any of the eigenforms. Expressing $f$ as a linear combination of the $h_{i}$, we have

$$
f=\sum_{i=1}^{d} a_{i}(f) h_{i}=h_{1}+\sum_{i=2}^{d} a_{i}(f) h_{i} .
$$

Since $T_{q}(x)$ is irreducible, it is immediate that not all of the coefficients $a_{q}\left(h_{2}\right)$, $\ldots, a_{q}\left(h_{d}\right)$ are 0 . Without loss of generality, we assume that $a_{q}\left(h_{2}\right) \neq 0$. By row reducing the Miller basis, we can obtain a basis $g_{1} \ldots g_{d}$ so that $a_{1}\left(g_{1}\right)=$ $1, a_{1}\left(g_{j}\right)=0$ for $j \neq 1$, and $a_{q}\left(g_{2}\right)=1, a_{q}\left(g_{j}\right)=0$ for $j \neq 2$, and $K\left(g_{j}\right)=\mathbb{Q}$ for every $g_{j}$.

Expressing $f$ in terms of the new basis, we realise that one of the Fourier coefficients $a_{q^{2}}\left(g_{3}\right), \ldots, a_{q^{2}}\left(g_{d}\right)$ must be non-zero. By repeating the row reduction argument and producing a new basis each time, we construct a basis $F_{1}, \ldots F_{d}$ with the property that $a_{q^{i-1}}\left(F_{j}\right)=\delta_{i, j}$. In addition, $K\left(F_{i}\right)=\mathbb{Q}$ for every $F_{i}$. Expressing $f$ in terms of this basis, and observing that $a_{q^{l}}(f) \in \mathbb{Q}\left(a_{q}(f)\right)$ for every $l \in \mathbb{Z}$, we have

$$
f=\sum_{i=1}^{d} a_{q^{i-1}}(f) F_{i}=\sum_{i=1}^{d} b_{i} F_{i} \text { where } b_{i} \in \mathbb{Q}\left(a_{q}\right) .
$$

Thus, for any $n, a_{n}(f) \in \mathbb{Q}\left(a_{q}(f)\right)$, and so the lemma follows.

Lemma 2.2. If $T_{q}(x)$ is irreducible for some prime $q$ with Galois group $G$, then for any other $n, T_{n}(x)$ has exactly one irreducible factor. In addition, if $G=S_{d}$, then the irreducible factor of $T_{n}$ has degree $d$ or 1 . 
Proof. Suppose $T_{q}(x)$ is irreducible with Galois group $G$. Then $G$ acts transitively on the roots of $T_{q}(x)$. Since the roots of $T_{n}(x)$ by Lemma 2.1, are rational linear combinations of the roots of $T_{q}(x)$, they form a Galois orbit with a $G$ action. Thus, they are all roots of the same irreducible polynomial. If, in addition, $G=S_{d}$, then the roots of $T_{n}(x)$ must form an orbit for a transitive $S_{d}$ action, and thus must all be equal, or all distinct. This proves the lemma.

Consider a Galois extension $L / \mathbb{Q}$ with Galois group $G$ discriminant $d_{L}$ and degree $n_{L}$. Suppose $S$ is the set of primes in $K$ ramified in $L$. Let $C$ be a collection of conjugacy classes in $G$. Let

$$
\pi_{C}(x)=\text { the number of primes } v \in \mathcal{O}_{L} ; N_{L / \mathbb{Q}}(v) \leq x \text { and } \text { Frob }_{v} \in C .
$$

The Chebotarev density theorem states that

$$
\pi_{C}(x) \sim \frac{|C|}{|G|} \pi(x)
$$

where $\pi(x)=$ the number of primes $p \in \mathbb{Z} ; p \leq x$.

The following unconditional effective version of this theorem was provided by Lagarias, Montgomery and Odlyzko in [7], we state a version due to Serre (see [11], Theorem 3, page 132).

Proposition 2.3. (Effective version of the Chebotarev density theorem) If $x \geq 3$ and $\left.\log x \geq c\left(\log d_{L}\right)\left(\log \log d_{L}\right)\left(\log \log \log 6 d_{L}\right)\right)$, then $\pi_{C}(x) \ll \frac{|C|}{|G|} \pi(x)$, where $c$ is an absolute constant.

We can bound the discriminant $d_{L}$ of $L$ by the following (see [11] Page 129, Proposition $4^{\prime}$ for a general statement)

Proposition 2.4. (Hensel) $\log d_{L} \leq\left(n_{L}-1\right) \sum_{l \in S} \log l+n_{L} \log n_{L}$.

\section{Fourier coefficients of Hecke eigenforms}

Let $f$ be a normalised Hecke eigenform, and suppose that for some $q$,

$$
\left[\mathbb{Q}\left(a_{q}(f)\right): \mathbb{Q}\right]=d \text {. }
$$

This is the same as saying $T_{q}(x)$ is irreducible. From Lemma 2.1, we know that $K(f)=\mathbb{Q}\left(a_{q}(f)\right)$.

Theorem 3.1. Let $L \subset K(f)$ be any proper subfield. Then,

$$
\sharp\left\{p \leq x ; a_{p}(f) \in L\right\} \ll \frac{x}{(\log x)^{1+\delta}},
$$

for some $\delta>0$.

Proof. Let $K(f)=K$, and let $L \subset K(f)$ be a proper subfield. Let $\lambda \in \mathcal{O}_{K}$ be a prime of degree $f \geq 2$ lying above $l \in \mathcal{O}_{L}$. By a well-known construction of Deligne and Serre (see [10], pages 260-261), there exists a continuous representation

$$
\rho_{f, \lambda}: \operatorname{Gal}(\overline{\mathbb{Q}} / \mathbb{Q}) \longrightarrow \mathrm{GL}_{2}\left(\mathcal{O}_{K} / \lambda\right)
$$

satisfying the following conditions for $p \neq l$ : 
(i) $\rho_{f, \lambda}$ is unramified at $p$

(ii) $\operatorname{trace}\left(\rho_{f, \lambda}\left(\operatorname{Frob}_{\pi}\right)\right)=a_{p}(f)$, for any prime ideal $\pi$ lying above $p$.

Let $S \subset \mathrm{GL}_{2}\left(\mathcal{O}_{K} / \lambda\right)$ be the set of elements whose trace lies in the subfield $\mathcal{O}_{L} / l \subset O_{K} / \lambda$. By a simple counting argument, we see that

$$
\sharp(S) \ll l^{3 f+1} \text { and } \sharp\left(\mathrm{GL}_{2}\left(\mathcal{O}_{K} / \lambda\right)\right) \sim l^{4 f} .
$$

Let $M$ be the fixed field of the kernel of the representation $\rho_{f, \lambda}$. By the effective version of the Chebotarev density theorem in $M$, if $l \sim(\log x)^{\delta}$, then

$$
\sharp\left\{p \leq x ; \operatorname{trace}\left(\rho_{f, \lambda}\left(\operatorname{Frob}_{p}\right)\right) \in \mathcal{O}_{L} / l \subset O_{K} / \lambda\right\} \ll \frac{\sharp(S)}{\sharp\left(\mathrm{GL}_{2}\left(\mathcal{O}_{K} / \lambda\right)\right)} x / \log x .
$$

By the bounds on $\sharp(S)$ and $\sharp\left(\mathrm{GL}_{2}\left(\mathcal{O}_{K} / \lambda\right)\right)$ and on $l$, we see that

$$
\sharp\left\{p \leq x ; \operatorname{trace}\left(\rho_{f, \lambda}\left(\operatorname{Frob}_{p}\right)\right) \in \mathcal{O}_{L} / l \subset O_{K} / \lambda\right\} \ll \frac{x}{l^{f-1} \log x} \ll \frac{x}{(\log x)^{1+\delta}} .
$$

This proves the theorem.

Proof of Theorem 1.1. By Lemma 2.2, if $T_{q}(x)$ is irreducible, then $T_{p}(x)$ is reducible if and only if it has a repeated root. Thus, $K\left(f_{1}\right)$ contains $\mathbb{Q}\left(a_{p}\left(f_{1}\right)\right)$ as a proper subfield. Since there are only finitely many proper subields of $K\left(f_{1}\right)$, we can apply Theorem 3.1 to each subfield. Thus, we see that

$$
\sharp\left\{p \leq x ; T_{p}(x) \text { is reducible }\right\} \ll \frac{x}{(\log x)^{1+\delta}} .
$$

This proves the theorem.

\section{Initial Fourier coefficients}

Proof of Theorem 1.2. By Lemma 2.2, we know that $T_{n}(x)$ is reducible only if it has a single repeated root, i.e., $a_{n}\left(f_{1}\right)=\cdots a_{n}\left(f_{d}\right)=a \in \mathbb{Z}$. Suppose this holds for some $i, 2 \leq i \leq d$. Let $h_{i}$ be as in Lemma 2.1. Let $h_{i}$ be written as a linear combination of the eigenforms as

$$
h_{i}=\sum_{i=1}^{d} c_{i, j} f_{j} .
$$

Since $a_{1}\left(h_{i}\right)=0$ and $a_{1}\left(f_{j}\right)=1$ for every $f_{j}$, we conclude that $\sum_{i=i}^{d} c_{i, j}=0$. Thus,

$$
1=a_{i}\left(h_{i}\right)=\sum_{i=1}^{d} c_{i, j} a_{i}\left(f_{j}\right)=\sum_{i=1}^{d} c_{i, j} a=0,
$$

which shows us that our assumption is false. This proves the theorem. 


\section{Comparison Theorems for Fourier coefficients of two eigenforms}

Let $f$ and $g$ be two distinct Hecke eigenforms for $\mathrm{SL}_{2}(\mathbb{Z})$ of weights $k_{1}$ and $k_{2}$ respectively. If $k_{1}=k_{2}$, then Theorem 1.1 implies the following:

Theorem 5.1. If $T_{q}(x)$ is irreducible for some prime $q$, then

for some $\delta>0$

$$
\sharp\left\{p \leq x ; a_{p}(f)=a_{p}(g)\right\} \ll \frac{x}{(\log x)^{1+\delta}}
$$

In the case $k_{1} \neq k_{2}$, we can prove a similar result unconditionally.

Theorem 5.2. If $k_{1} \neq k_{2}$, then

$$
\sharp\left\{p \leq x ; a_{p}(f)=a_{p}(g)\right\} \ll \frac{x}{(\log x)^{1+\delta}}
$$

for some $\delta>0$

In [10], section 5, Ribet studied pairs of Galois representations and showed that if $l$ is sufficiently large, the two Galois representations $\rho_{f, \lambda}$ and $\rho_{g, \lambda}$ are "as independent as possible", i.e., the image of the product representation is as large as possible. In the case $f$ and $g$ are as in Theorem 5.2, we state Ribet's theorem as follows:

Lemma 5.3. $\operatorname{Im}\left(\rho_{f, \lambda}, \rho_{g, \lambda}\right)=$ $\left\{\left(u, u^{\prime}\right) \in G L_{2}\left(\mathcal{O}_{\lambda}\right) \times G L_{2}\left(\mathcal{O}_{\lambda}\right) ; \operatorname{det}(u)=v^{k_{1}-1} ; \operatorname{det}\left(u^{\prime}\right)=v^{k_{2}-1}, v \in \mathcal{O}_{\lambda}\right\}$

Let $\mathbf{F}_{\lambda}=\mathcal{O}_{\lambda} / \lambda$. If we let $\bar{\rho}_{f, \lambda}: \operatorname{Gal}(\overline{\mathbb{Q}} / \mathbb{Q}) \longrightarrow \mathrm{GL}_{2}\left(\mathbf{F}_{\lambda}\right)$ denote the residual representation of $\rho_{f, \lambda}$, we see that

$$
\begin{gathered}
\operatorname{Im}\left(\bar{\rho}_{f, \lambda}, \bar{\rho}_{g, \lambda}\right)= \\
\left\{\left(u, u^{\prime}\right) \in \mathrm{GL}_{2}\left(\mathbf{F}_{\lambda}\right) \times \mathrm{GL}_{2}\left(\mathbf{F}_{\lambda}\right) ; \operatorname{det}(u)=v^{k_{1}-1} ; \operatorname{det}\left(u^{\prime}\right)=v^{k_{2}-1}, v \in \mathbf{F}_{\lambda}\right\} .
\end{gathered}
$$

Proof of Theorem 5.2. Let $E / \mathbb{Q}$ be an extension containing both $E_{f}$ and $E_{g}$, and $\lambda$ a degree one prime in $E$ of norm $l$ (by the prime number theorem in number fields, we know that degree 1 primes are of full density, see [6], Theorem 4, Page 350). Let

$$
\begin{gathered}
S=\left\{\left(u, u^{\prime}\right) \in \mathrm{GL}_{2}\left(\mathbf{F}_{\lambda}\right) \times \mathrm{GL}_{2}\left(\mathbf{F}_{\lambda}\right) ; \operatorname{det}(u)=v^{k_{1}-1}, \operatorname{det}\left(u^{\prime}\right)=v^{k_{2}-1}, v \in \mathbf{F}_{\lambda}\right\}, \\
S^{\prime}=\left\{\left(u, u^{\prime}\right) \in S ; \operatorname{trace}(u)=\operatorname{trace}\left(u^{\prime}\right)\right\}, \\
|S| \leq l^{7} \text { and }\left|S^{\prime}\right| /|S| \leq 1 / l .
\end{gathered}
$$

By the above, we know that the image of the product representation $\bar{\rho}_{f, \lambda} \times \bar{\rho}_{g, \lambda}$ is exactly the group $S$. Let $K$ be the fixed field of the kernel of the representation, i.e., $\operatorname{Gal}(K / \mathbb{Q})=S$. Then, by the calculation in (a) above, the proportion of elements of $\operatorname{Gal}(K / \mathbb{Q})$ whose image lies in $S^{\prime}$ is approximately $1 / l$.

Let $C$ denote the set $\left\{\right.$ Frob $\left._{\pi}\right\}$ for primes $\pi \in K$ with $\bar{\rho}_{f, \lambda} \times \bar{\rho}_{g, \lambda}\left(\right.$ Frob $\left._{\pi}\right) \in$ $S^{\prime}$. Then $C$ is clearly invariant under conjugation, and thus we can apply the 
effective version of the Chebotarev density theorem, Proposition 2.3. We see that $|C| /|\operatorname{Gal}(K / \mathbb{Q})|=\left|S^{\prime}\right| /|S|$ and thus,

$$
\pi_{C}(x) \ll \frac{\left|S^{\prime}\right|}{|S|} \pi(x) .
$$

By property (ii) of the Galois representations $\rho_{f, \lambda}$ and $\rho_{g, \lambda}$, we see that

$$
\sharp\left\{p \leq x ; a_{p}(f)=a_{p}(g)\right\} \ll \sharp\left\{p \leq x ; \operatorname{Frob}_{\pi} \in C\right\},
$$

for some $\pi$ dividing $p$. Thus, we see that

$$
\sharp\left\{p \leq x ; a_{p}(f)=a_{p}(g)\right\} \ll \frac{\left|S^{\prime}\right|}{|S|} \pi(x)
$$

if $x$ is sufficiently large. By property (i) of the representations, we know that $K$ is ramified only at $l$, and so we can apply Proposition 2.4. Using equation 1, we see that

$$
\log d_{L} \leq 8 l^{7} \log l
$$

Thus, for large $x$, if we choose $l \sim(\log x)^{1 / 8}$, we see that the conditions of Proposition 2.3 are satisfied, and so we have, by all of the above,

$$
\sharp\left\{p \leq x ; \operatorname{Frob}_{\pi} \in C\right\} \ll \frac{1}{l} \pi(x) \ll x /(\log x)^{9 / 8} .
$$

This proves the theorem.

\section{Conditional estimates}

The estimates in Theorem 1.1 and Theorem 5.2 can be improved considerably if we assume the generalised Riemann hypothesis for Dedeking Zeta functions of number fields (GRH) ( see [9]). By the methods of [9], it follows that we have the following results.

Theorem 6.1. Assume GRH for Dedekind zeta functions of number fields. If $T_{q}(x)$ is irreducible for some prime $q$, then

$$
\sharp\left\{p \leq x ; T_{p}(x) \text { is reducible }\right\} \ll x^{1-\delta}
$$

for some $\delta>0$.

Theorem 6.2. Assume GRH for Dedekind Zeta functions of number fields. If $k_{1} \neq k_{2}$, then

$$
\sharp\left\{p \leq x ; a_{p}(f)=a_{p}(g)\right\} \ll x^{1-\delta}
$$

for some $\delta>0$. 


\section{References}

[1] K. Buzzard, On the eigenvalues of the Hecke operator $T_{2}$, J. Number Theory 57 (1996), 130-132.

[2] J.B. Conrey, D.W. Farmer, P. J. Wallace, Factoring Hecke polynomials modulo a prime, Pacific J. Math. 196 (2000), 123-130.

[3] D. W. Farmer, K. James, The irreducibility of some level-1 Hecke polynomials, Math Comp, 71 (2002) 1263-1270.

[4] K. James, K. Ono, A note on the irreducibility of Hecke polynomials, J. Number Theory 73 (1998), 527-532.

[5] S. Lang, Introduction to modular forms, Grundlehren der mathematischen Wissenschaften, No. 222. Springer-Verlag, Berlin-New York, 1976.

[6] _ Algebraic number theory, Second edition. Graduate Texts in Mathematics, 110. Springer-Verlag, New York, 1994.

[7] J. C. Lagarias, H. L. Montgomery, A. M. Odlyzko, A bound for the least prime ideal in the Chebotarev density theorem, Invent. Math. 54 (1979), 271-296.

[8] H. Hida, Y. Maeda, Non-abelian base change for totally real fields, Olga Taussky-Todd: in memoriam. Pacific J. Math. (1997), Special Issue, 189-217.

[9] M.R. Murty, V.K. Murty, N. Saradha, Modular forms and the Chebotarev density theorem, Amer. J. Math. 110 (1988), 253-281.

[10] K. Ribet, On l-adic representations attached to modular forms, Invent. Math. 28 (1975), 245-275.

[11] J.-P. Serre, Quelques applications du théorème de densité de Chebotarev, (French) [Some applications of the Chebotarev density theorem] Inst. Hautes Études Sci. Publ. Math. No. 54, (1981), 323-401.

[12] W. Stein, www.modular.fas.harvard.edu/ stein

Max Planck Institut für Mathematik, Vivatsgasse 7, D-53111, Bonn, Germany.

E-mail address: sbaba@mpim-bonn.mpg.de

Department of Mathematics and Statistics, Queen's University, Kingston, OnTARIO, K7L 3N6, CANADA.

E-mail address: murty@mast.queensu.ca 